\title{
Engineering the Stem Cell Niche and the Differentiative Micro- and Macroenvironment: Technologies and Tools for Applying Biochemical, Physical and Structural Stimuli and Their Effects on Stem Cells
}

\section{Paolo Di Nardo, Marilena Minieri, and Arti Ahluwalia}

\begin{abstract}
In recent years there has been an explosion of interest in stem cell research, given their promising medical applications in cell-based tissue regeneration, drug testing and of course basic research. A decade of restless experimental and clinical research has demonstrated that the routine use of stem cells to repair solid organs is not at hand in spite of recent excessively enthusiastic announcements in the press and even serious scientific journals. Indeed, biologists only partially comprehend cell-differentiating mechanisms and have mapped only a few of the extrinsic and intrinsic factors involved. Even less is understood the complex qualitative, quantitative and temporal orchestration of these factors in the different steps featuring the whole differentiating process.

Most of the current research is centred on the identification of soluble ligands which regulate and control signalling pathways, and our knowledge on the role of the physical and structural microenvironment is still scarce. In this chapter, we focus only on cues which can be controlled externally using mechanical and structural parameters, and so can be easily defined using appropriate engineering and design. Firstly, the influence of the single parameters on cell behaviour is described, and then we discuss how technological tools such as biomaterials, scaffolds and bioreactors, as well as well-constructed and defined multiscale classification models can be best employed to engineer artificial biomimetic in vitro systems.
\end{abstract}

Keywords Engineering $\cdot$ Microenvironment $\cdot$ Stem cells $\cdot$ Stimuli

"Da mihi ubi consistam,... et terram caelumque movebo" Give me where to stand. . and I will move the earth

(Archimedes, 287 b.c.).

\footnotetext{
A. Ahluwalia $(\bowtie)$

Interdepartmental Research Centre "E. Piaggio", University of Pisa, Pisa, Italy

e-mail: arti.ahluwalia@centropiaggio.unipi.it
} 


\section{Introduction}

Stem cells are inherently unstable and their fate in vivo is modulated by the socalled niche, i.e. an anatomic and functional unit in which the unstable stem cells are confined in a semi-quiescent state. Upon specific requirements to preserve tissue integrity and function, stem cells asymmetrically divide and the daughter cells leave the niche towards a new decoy environment directing natural stem cell instability in favour of the acceptance of the required phenotype. It is generally accepted that it is the "symmetry" or "featurelessness" of the niche microenvironment which maintains an undifferentiated status in stem cells and perturbations are either self-generated due to instabilities or arise from external factors. This is certainly true of the embryo in the first stages of development. Some external signals or non-linear perturbations must lead to non-symmetry of the system to pull it out of quiescent stemness $[1,2]$. Therefore, stem cells are in a state of metastable equilibrium within and without their niches, and this has led to a huge number of papers fervently reporting on the new factors which drive stem cell differentiation. The past few years have been spent in a futile attempt to identify "the soluble factor" (growth factor, cytokine, chemical, etc.) able to spark gene programmes leading stem cells to adopt different phenotypes. The injection of one or a mixture of soluble factors into the diseased organ in order to recruit, expand and differentiate the rare stem cells available to substitute damaged cells has even been suggested [3]. Most of this fervour is driven by the promise of tissue regeneration by stem cells. In fact the field of tissue engineering has undergone a definite shift towards the explicit use of stem cells, although few recognize that it is these cells that are largely responsible for the success of engineered skin, blood vessels and the few others we may care to count on one hand. Figure 1 shows how the fraction of papers explicitly dealing with stem cells in the journal Tissue Engineering has changed since it was first established in 1995 . Over $35 \%$ of papers now focus on the factors that guide stem cell differentiation and the numbers are certain to increase. The general impression that one gains from the current literature is that anything will make mesenchymal stem cells (MSCs) or bone marrow stem cells (BMSCs) as well as embryonic stem cells (ESCs) or embryoid bodies differentiate, and it is highly likely that the very act of isolating them and plating in vitro causes changes in their phenotype.

The attempt to solve extremely complex problems with oversimplistic solutions generated inconsistent results that, however, were able to further fuel hopes in patients in which conventional treatments were no longer able to counteract their diseases. A mindful survey of the current literature on cell differentiation processes ought to have suggested a more careful and articulated approach to stem cell manipulation and clinical application.

Indeed, the territory of stem cell biology and engineering is still riven with pitfalls and obstacles - and more importantly blind spots. We face a huge number of technological tasks before being able to gain a mechanistic understanding of stem cell destiny in vitro. Without a basic comprehension of the mechanisms, trying to control stem cell destiny will get us nowhere. And the number of papers published 


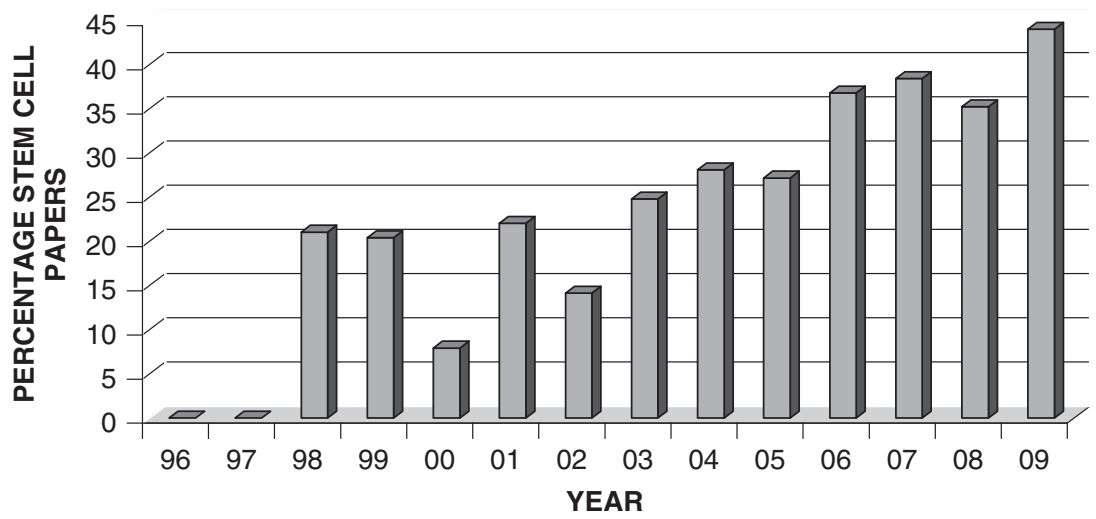

Fig. 1 Percentage of papers dedicated to stem cells in the journal Tissue Engineering since its inception in 1995. By the end of this decade, $50 \%$ of all papers in regenerative medicine will probably focus on the use of stem cells

on the subject, often presenting conflicting results, is a strong evidence that so far the research has been leading up blind alleys.

We need to find solutions to innumerable biological and technical problems and the majority of these are related to three main issues:

- Stem cell source. There is a scarcity of stem cells in the adult body. Often they are difficult to identify, locate and isolate.

- Control of stemness in vitro. The cells should ideally be amplified in vitro, but only in such a way so as to maintain symmetry: that is, each daughter is an identical copy of the mother.

- Control of differentiative status. The cells need to be differentiated in a controlled manner without becoming tumoural.

Can we surmount these? The question of stem cell source is intricately linked with the question of aging and the role of stem cells in tissue homeostasis and repair as well as with the natural limits of reparative potential of these cells and the human body in general. While blood cells, skin and epithelial tissue in general have a high turnover and therefore an abundant supply of stem cells, in other tissues such as the liver and pancreas, homeostasis is maintained by adult cells which can proliferate to a certain extent and a small number of resident stem cells are called into action in injury. In those tissues with low cell turnover and low regenerative potential, such as the heart and brain, adult or autologous sources are probably out of the question, since as illustrated in Fig. 2, they have very few if any stem cells [4]. Here, the reparative capacity is limited and homeostasis is maintained by differentiated cells. As far as the other two issues mentioned are concerned, keeping in mind the capacity and limits of intrinsic self-renewal amongst specific tissues, there is little doubt that the key lies in developing a mechanistic understanding of the influence of the microenvironment on cell behaviour and function at multiple levels [5]. 


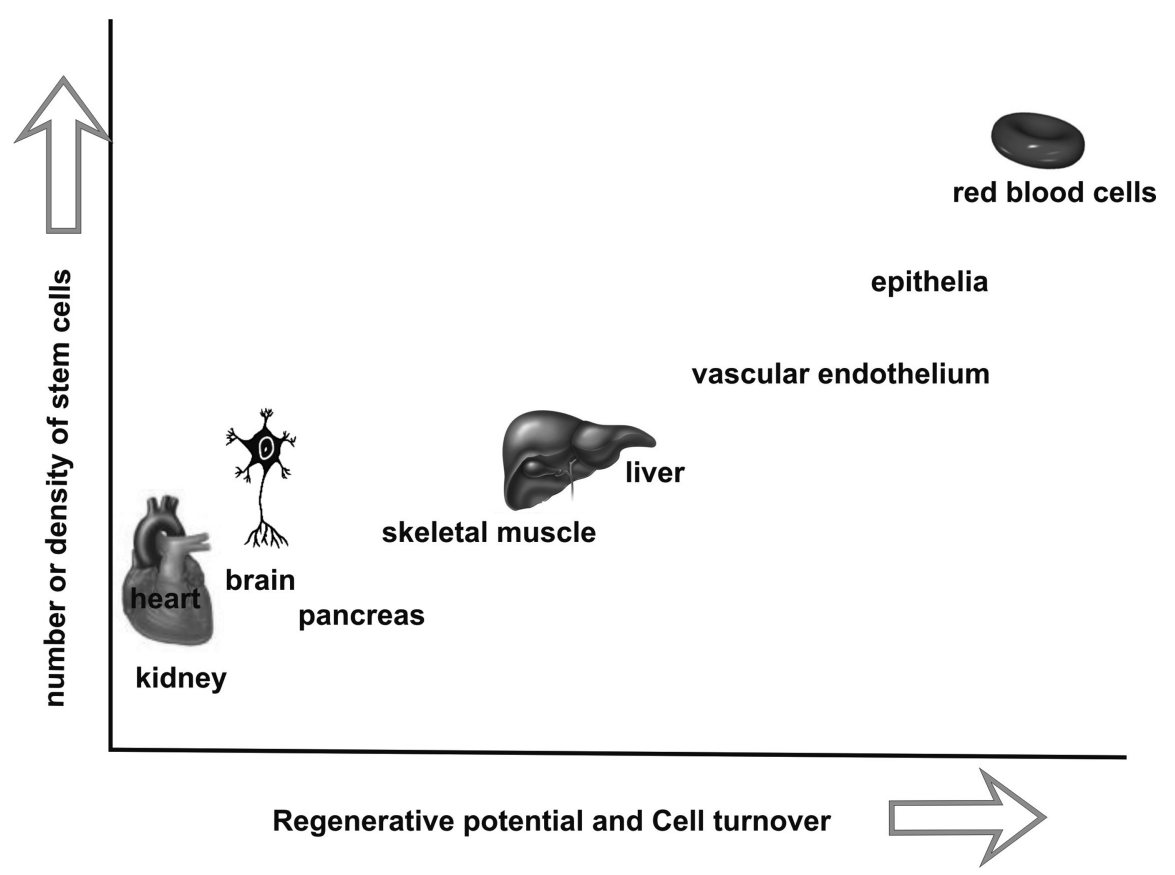

Fig. 2 The possibility of isolating stem cells from adult tissue depends on their density, which in turn depends on the regenerative potential of the tissue in question

\section{The Micro- and Macroenvironment}

Three principal extrinsic cues are known to guide cell function and fate: the biochemical microenvironment, including ligands, signalling molecules and other cells, the physico-chemical environment which comprises gradient-dependent factors such as surface properties, oxygen tension, $\mathrm{pH}$ and temperature, and finally the mechano-structural environment. The mechano-structural environment is the architecture in two and three dimensions as well as mechanical forces such as stress and strain, all of which act in a non-linear but fairly constant manner. Together the three classes of stimuli represent what we define as the tripartite axes of cues. Superimposed upon this is the one and only linear factor which acts on cells and which we have no control over: Time, with a capital T. Figure 3 illustrates this concept, and as shown the number of possible variables is almost infinite.

Cells in vivo are surrounded by all these cues together in the microenvironment in the form of the prestressed extracellular matrix, prestressed neighbouring cells, endocrine and paracrine signals, blood flow, body movement and forces and nutrient diffusion. Furthermore, cells themselves will remodel and modulate their own habitat, an aspect which is often ignored in in vitro experiments, as are most of the mechano-structural stimuli. 

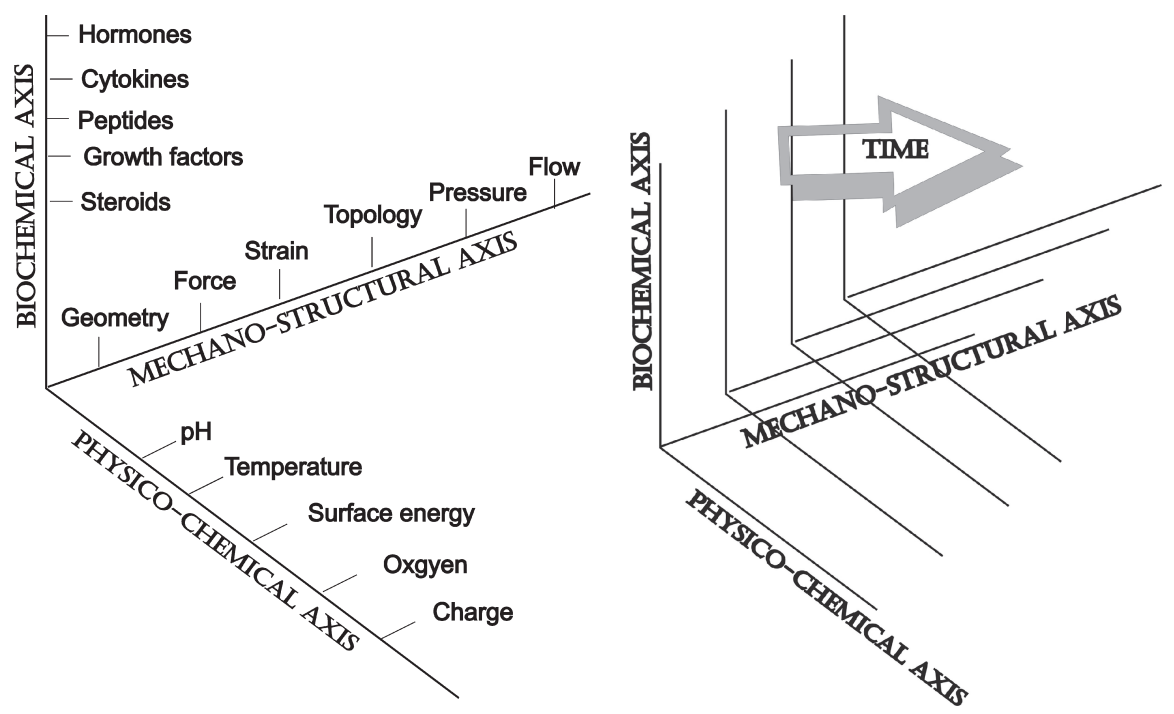

Fig. 3 The tripartite axes of cues which act on cells. (a) The three principal axes must be referred to a fourth axis Time, which moves inexorably forward in a linear manner. Although we have no control over Time, "the biological clock", it must be taken into consideration. (b) Each of the principal axes is composed of a subset of factors, and their "intensity" influences cell function in a non-linear manner

Fig. 4 Both extrinsic and intrinsic factors influence the orchestration of cellular function. Note that there is an interdependency between the two
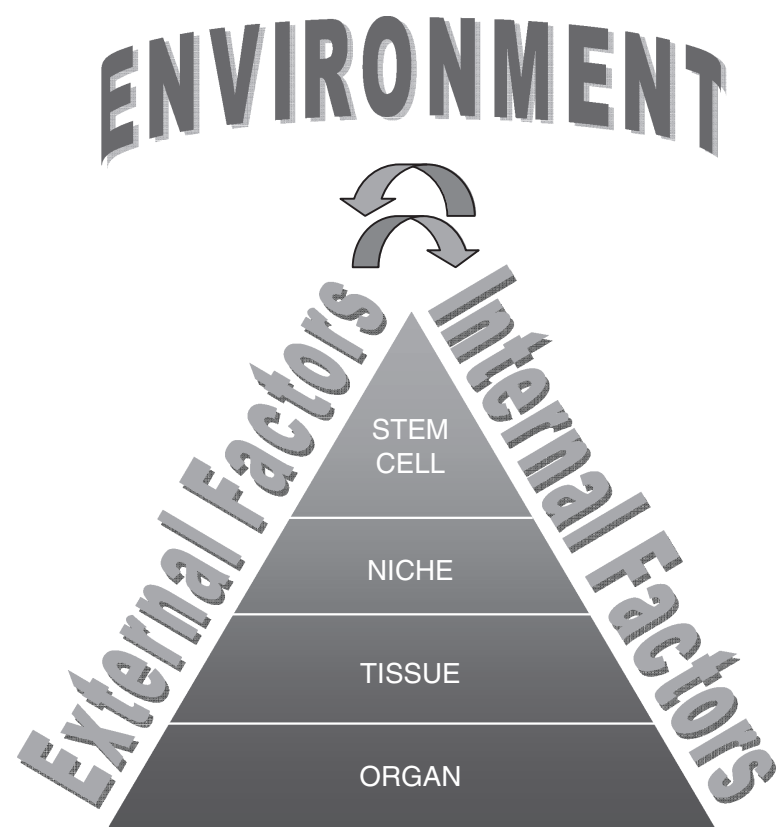
In Fig. 4 we schematize the hierarchic organization of the stem cell habitat. All cells coordinate their behaviour and function in response to signals from the macroto the microscale, within and without their tissues. The signals are not necessarily soluble ligands but can be external or internal.

\section{Time}

Time is probably one of the most important but least considered variables which determine stem cell fate. Even stem cells are equipped with a biological clock which ticks constantly. Therefore, unless the tick frequency is decreased by techniques such as freezing or hypoxic conditions, the functional ability of stem cells decreases with their age [6]. There is however some question as to whether the aging is due to intrinsic aging of the stem cells or due to the aging microenvironment of the organism itself, since aged skeletal progenitor stem cells are as effective as young ones when implanted in young animals. According to Rando [4] the extent to which aging deteriorates the stem cell or its microenvironment depends on the regenerative potential of stem cells (see Fig. 2). The implication is that stem cells with a high density and regenerative potential age intrinsically, due to the Hayflick limit or "replicative senescence", whereas those resident in tissues with low cellular turnover are influenced by senescence of the microenvironment.

\section{Biochemical Microenvironment}

In the human body, biochemical cues are generally provided by soluble ligands which may be secreted by paracrinal cells or supplied by a capillary network. Insoluble ligands are also present; these are adhesion proteins or molecules such as collagen, laminin and carbohydrates. They are considered insoluble because they have very small diffusion constants. Other insoluble cues arise from neighbouring cells themselves, through adhesive and cohesive junctions. The recognition that cells themselves remodulate their extracellular milieu is of crucial importance in all aspects of cell biology, and particularly in regenerative medicine and engineering. Much of the work on stem cell biology to date has focused on the use of a handful of soluble factors in vitro to differentiate cells. In most cases the signalling pathways are still not clearly identified and methods used to differentiate cells are determined empirically on the basis of trial and error. For example, human or mouse embryonic stem cells can be coerced to differentiate into neuron-like, pancreatic $\beta$-cell-like, adipose-like, hepatocyte-like and osteoblast-like cells by the addition of substances such as dexamethasone, retinoic acid and dimethyl sulphoxide [7] or by gene transfection with transcription factors such as osterix [8]. Clearly, none of these routes are clinically acceptable or in anyway controllable. Likewise, bone marrowor adipose tissue-derived mesenchymal stem cells can be differentiated into osteocytes, chondrocytes or adipocytes by the addition of dexamethasone, ascorbic acid, 
TGF- $\beta$ and indomethacin [9]. Differentiation into cells such as neurons, hepatocytes and cardiomyocytes has also been reported $[10,11]$, although several investigators also provide more recent data, in which mesenchymal stem cell plasticity is disproved $[12,13]$. The type of trial-and-error studies conducted in the past 20 years should have induced investigators to probe the spectrum of biochemical stimuli in the stem cell microenvironment more profoundly but so far has only led to great confusion. The story of cardiac stem cells is a case in point; investigators have passively accepted the concept that stem cell fate is mostly governed by soluble factors (growth factors/cytokines, epigenetic factors, etc.). As a consequence, the in vitro replication of niche conditions is focused on the identification of a mixture of soluble factors. Indeed, attempts at activating both in vitro and in vivo stem cell lineage specification have been based on the assumption that a few soluble factors, sometimes injected into either the bloodstream or the damaged tissue, can govern the whole process leading stem cells to full differentiation, i.e. stem cell specification and determination, sarcomere assembly, and cardiomyocyte integration into the myocardial architecture $[14,15]$. To this end, only a relatively small number of soluble factors have been investigated to verify their actual potential in inducing cardiac stem cell differentiation into cardiomyocytes. These factors pertain to two distinct categories: biochemical and epigenetic factors. Biochemical factors, such as IGF-1, HGF, oxytocin, TGF- $\beta 1$ and FGF-2, added in different concentrations in stem cell culture media have induced the expression of early and late cardiac markers, but the demonstration of a fully controlled differentiation into cardiomyocytes is still lacking [16]. Similar results have also been obtained with epigenetic modulators regulating chromatin stability and gene expression. Chromatin modifications are important for several nuclear processes, including DNA repair, replication, transcription and recombination, biological processes that probably change with Time or age.

Given the complexity of the biochemical microenvironment, in our opinion the logical way to define a niche or a differentiative microenvironment is by the delivery of tissue-specific stimuli through an in vitro system that recapitulates the in vivo functions of a natural niche. In this way, cells are provided a biomimetic habitat in which stem cell fate, e.g. self-renewal versus differentiation, can be controlled in a defined and, to a large extent, predictable manner through the provision of cellcell and soluble-mediator cross talk through induction by tissue-specific cells [17]. In fact, distinct cell types (e.g. stromal cells and endothelial cells) and the extracellular matrix [18] are major players in the regulation of stem cells within their niche. Recapitulating this environment requires innovative and infinitely flexible cell culture systems in which the presence of feeder cells, tissues or appropriate ligands in specific space- and time-dependent configurations can be combined in a tightly controlled manner to provide a wide array of multi-parametric signals which can be tailored to guide stem cell fate, leading to new paradigms in stem cell culture. One of the authors has already proposed and patented such a system, the MCB (multicompartmental bioreactor), which is schematized in Fig. 5 with specific reference to the differentiation of stem cells for cardiac regeneration [19]. 


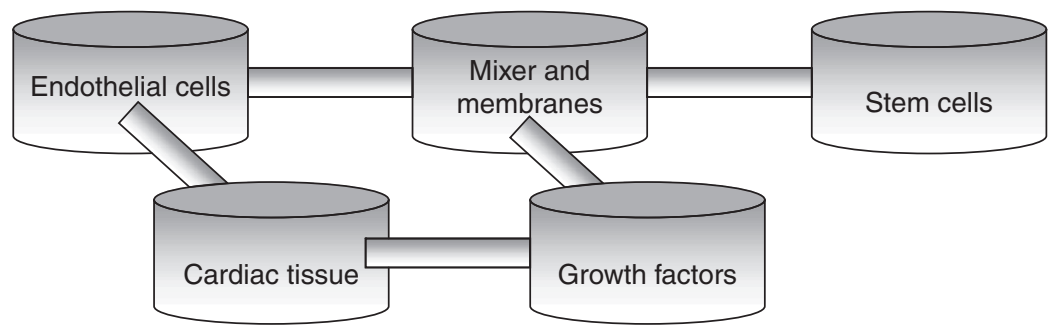

Fig. 5 Concept of the MCB (multicompartmental bioreactor) for providing tissue-specific biochemical and physical cues for cardiac tissue regeneration in vitro. Here, the biochemical cues are provided by the tissues and cells which condition cardiac habitat in vivo. The biochemical cues are vehicled to the mixer and can be sorted and conveyed to the stem cells through membranes with custom pore size. Physical and structural cues can be supplied by three-dimensional scaffolds, flow and hydrodynamic or compressive forces

\section{Physico-chemical and Mechano-structural Axes - The Macroenvironment}

Several studies have suggested the potential effects of other non-soluble stimuli, such as those induced by physical factors, on cell differentiation processes. So far at least 10 different non-biochemical cues which influence cell phenotype have been identified and new ones are constantly being added. The stimuli known to influence stem cell behaviour and fate are illustrated in Fig. 6. Most of these are the external factors noted in Fig. 4, whilst others are internal, but modulated somehow by the external environment. For example, the oxygen concentration will depend firstly

Fig. 6 The multitude of physico-chemical and mechano-structural cues known to influence stem cell fate. From the figure we can distinguish the physico-chemical cues $(\mathrm{pH}$, oxygen, surface energy, temperature and electromagnetic fields) from the mechano-structural factors (topology, gravity, etc.) and Time which stands on its own and is unidirectional

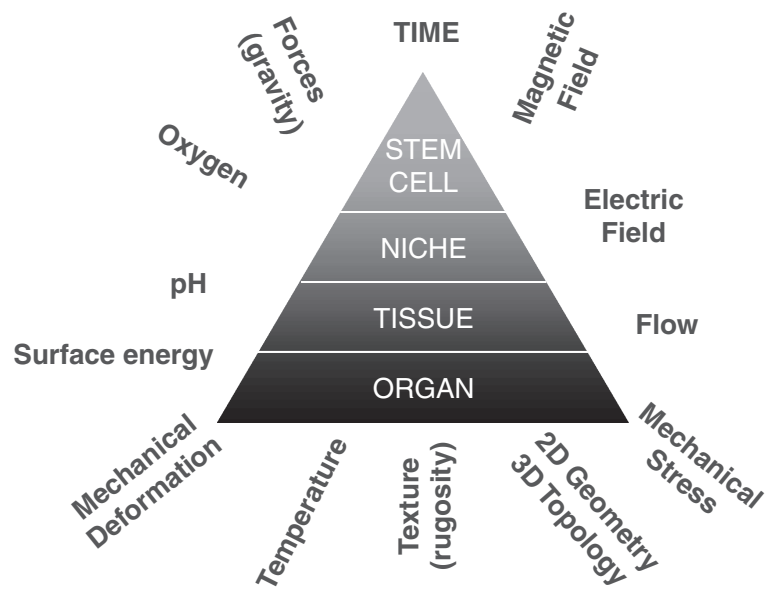


on atmospheric partial pressure and then on the distance between cells and oxygen source (capillaries).

Numerous recent papers have sprouted showing how even minor experimental modifications can change cell phenotype. Indeed, stem cells are so very sensitive and unstable that even cell seeding density and seeding protocol have been observed to influence cell shape and gene expression [20]. Note, however, that seeding density is well known to influence shape and spreading of even adult cells and many cells only express specific genes and proteins when at confluence. Similarly, wettability has long been known to play a role in cell adhesion, but only in the short term until cells themselves modulate their extracellular environment [21], and recent reports show that the same effects are observed on stem cells [22]. In fact, Liu et al. report that in a foetal osteoblastic cell line, spreading and initiation of the differentiating cascade depends on surface energy, but over long timescales, the cells do converge to the same phenotype independent of the initial surface energy.

\section{Physico-chemical Factors}

Physico-chemical factors typically influence the cell microenvironment in a concentration-, field- or gradient-dependent manner. This includes chemical gradients, temperature gradients and time-varying electric or magnetic fields.

Probably one of the best known physico-chemical cues is oxygen concentration. In incubators, the acid-base balance is strictly regulated through the use of buffers, which ensure that the $\mathrm{pH}$ and $\mathrm{CO}_{2}$ tension remain similar to those in vivo. On the other hand, typical oxygen concentrations in vivo vary from 12.5 to $5 \%$, whilst in cell culture incubators, the oxygen concentration is the same as that in air, or $20 \%$. It is important to realize that while the atmospheric oxygen tension is relatively straightforward to control in hypoxic incubators, the actual oxygen tension at the cell surface will vary greatly from one experiment to the other because of the low solubility of oxygen. It will depend particularly on the height of the medium and the thickness of any construct used. Several reports show that lowered oxygen concentrations (5\%) increase stem cell proliferation [23-25]. Grayson et al. [26] have shown that even lower oxygen concentrations of about $2 \%$ increase MSC proliferation whilst maintaining an undifferentiated state, thus suggesting that hypoxic conditions are characteristic of the niche environment. Some authors have observed an induction of adipose-like phenotype in MSCs in severe hypoxia (1\%) [27], whilst others note that adipogenesis is suppressed at $6 \%$ oxygen with respect to $20 \%$ oxygen [28]. Lennon et al. report that rat MSCs exposed to 5\% oxygen during amplification show enhanced osteogenesis after implantation, compared with cells amplified in $20 \%$, and this is probably due to increased proliferation as suggested above [29]. More sophisticated oxygen sensing at the cell surface is mandatory for ensuring adequate control of this critical parameter and micro-pH sensors should be integrated into any bioreactor system for stem cell engineering. It is also useful to model oxygen consumption and gradients in stem cell culture systems using finite-element modelling tools. 
Temperature is also known to play a role in modulating stem cell behaviour. In particular, at lower temperature $\left(32^{\circ} \mathrm{C}\right), \mathrm{MSC}$ from young rats display reduced apoptosis and proteasome activity as well as increased expression of heat-shock proteins [30]. The same authors also observed that at reduced temperatures, the stemness of MSCs is better maintained as they may be inducing a quiescent state in the cells and protecting them against spontaneous differentiation [31]. Note that both reduced temperature and hypoxia also slow down the biological clock.

Surface tension, wettability and contact angle all are expressions of the interfacial or surface energy of a solid-liquid or solid-air interface. The surface energy can be changed by modulating the chemistry of a substrate. Studies on the interactions between cells and surfaces and proteins and surfaces date back to the 1970s, and it has been widely observed that cell adhesion and function is better on hydrophilic surfaces than on hydrophobic ones [32]. It is also known that cell adhesion is mediated by proteins. Furthermore, there is a critical limit of contact angle, $20^{\circ}$, below which no adhesion will occur because the surface is too hydrophilic and a superficial layer of water prevents protein adhesion. Curran et al. [33] show that stem cell differentiation is guided by surface chemistry and energy, independent of inductive media. Although all the surfaces tested maintained cell viability, silanized hydrophobic surfaces with $\mathrm{CH}_{3}$ end groups (so low surface energy) maintain MSC phenotype, while increasing the surface energy by adding $\mathrm{NH}_{2}$ - or $\mathrm{SH}$-terminal groups promotes osteogenesis. Further increase of surface energy by the addition of $\mathrm{OH}$ or $\mathrm{COOH}$ moieties promotes chondrogenesis.

Electric, magnetic and ultrasound fields are routinely used for therapeutic purposes, particularly for bone, cartilage and muscle injury, even though the mechanisms of wound healing and regeneration under these fields are not clearly understood. In this context, the cardiomyocyte and myocyte models are probably the most well known, since electrical stimulation ( $1 \mathrm{~Hz}, 5 \mathrm{~V} / \mathrm{cm}, 2 \mathrm{~ms}$ pulses) induces contraction coupling and structural organization of cardiomyocytes and smooth muscle cells [34]. Almost 20 years ago, Sauer et al. [35] demonstrated that a single 1990s burst of electromagnetic stimulation $(5 \mathrm{~V} / \mathrm{cm})$ of ESCs led to differentiation towards a cardiomyocyte phenotype. The same authors show that treatment of ESCderived embryoid bodies with field strengths ranging from 2.5 to $7.5 \mathrm{~V} / \mathrm{cm}$, applied only for the 1960s, dose-dependently increased differentiation towards the endothelial phenotype [36]. These findings highlight the acute sensitivity of embryonic stem cells as well as the need for more rigorous and standardized experimental protocols. Endothelial progenitor cells and muscle precursor cells can also be stimulated by electromagnetic fields to promote myocyte differentiation [37, 38]. Interestingly, electrical stimulation (10-40 V, $5 \mathrm{~ms}, 0.5 \mathrm{~Hz}$ pulses) of human embryonic fibroblasts was shown to cause loss of cell proliferation and cell number but also led to differentiation of fibroblasts into multinucleated myotube-like structures [39].

Weak magnetic pulses with higher frequency $(30-120 \mathrm{~Hz}, 1.25 \mathrm{mT}$ ) have been used to differentiate preosteoblastic cells [40], and the authors found that the fields affected only undifferentiated cells, not differentiated osteoblasts, indicating that the former were more sensitive. The increased sensitivity also leads to cell damage at high frequencies; for example, bone marrow-derived stem cells 
under high-frequency $(50 \mathrm{~Hz})$ and low-intensity magnetic $(0.8 \mathrm{mT})$ fields showed a reduction in the proliferation and differentiation of the granulocyte-macrophage progenitor (CFU-GM) compared to non-exposed bone marrow cells [41], while ESC which did not possess tumour suppressor genes showed adverse effects under magnetic fields of intensity $2.3 \mathrm{mT}$ at a frequency of $50 \mathrm{~Hz}$ [42]. Ultrasound has also been shown to induce differentiation. Finally in low-intensity ultrasound field studies, MSCs differentiate towards a chondrocytic phenotype [43, 44].

\section{Mechano-structural Microenvironment}

As represented in Fig. 3, the mechano-structural axis comprises those cues which condition the static nature of the cell habitat. Here we include 3D architecture, surface physical features such as roughness, bulk properties such as elastic modulus, as well as stress, strain and force. The most striking and repeatable differences are found between two- and three-dimensional (3D) environments. In fact, there is a dramatic change in habitat when cells are removed from an in vivo context to a Petri dish, and in particular, stem cells find themselves in a highly non-symmetrical context. Therefore, several reports have shown that encapsulated cells or spheroids are capable of maintaining stemness, because the microenvironment does not condition the cells to express differentiated proteins for coupling to an extracellular environment. Although there are few systematic studies on the comparative effects of 2D and 3D structures, there are clear indications that cells respond quite differently to the 3D environment which allows greater cell-cell interaction and maintenance of spherical morphologies. In this context, hydrogels such as those derived from alginate, collagen and hyaluronic acid have been shown to be quite promising - they provide a homogeneous, structureless soft $3 \mathrm{D}$ environment which is probably ideal for stem cell proliferation and maintenance, as well as for differentiation into softer tissues such as neural or hepatic $[45,46]$.

Three-dimensional environments can also provide more controlled spatial information to cells if they are architectured using techniques such as microfabrication based on rapid prototyping. In this case, the $3 \mathrm{D}$ system is usually nominated as a "scaffold" and provides a rigid and porous framework for cells to adhere on and spread in three dimensions. One of the first reports on architectured scaffolds employed for stem cell engineering describes a random pore (250$500 \mu \mathrm{m}$ ) salt-leached synthetic polymer scaffold [a blend of polylactide (PL) and polylactide- $\mathrm{co}$-glycolide (PLGA)] seeded with ESCs [47]. The cells generated complex capillary-like organized features which cannot be formed in a $2 \mathrm{D}$ environment. Moreover, the porous scaffold permitted the organization and orientation of ESCs, whereas a homogeneous isotropic soft gel did not, suggesting that both stiffness and a structured topology are important features to which the cells respond.

Further studies by Liu and Roy [48, 49] also confirmed that a porous rigid scaffold (tantalum in this case) promotes differentiation of ESCs into hematopoietic cells with respect to classical 2D cultures. Here, the cells were observed to interact with the scaffolds forming smaller aggregates, and thus increasing cell-substrate 
rather than cell-cell contact, probably led to increased expression of ECM and adhesion proteins. Similar results for porous PLGA scaffolds have been observed for ESC osteogenesis [16].

An important aspect of the mechano-structural environment is the chemical nature of the substrate, that is, the nature of the biomaterial used as an interface for cell adhesion and a structural support for tissues. Recent work has demonstrated that biomaterials, i.e. matrices, scaffolds and culture substrates, can present key regulatory signals to create artificial surrogate microenvironments that control stem cell fate. Although little is known about the influence of specific biomaterial features, factors, such as ligand density, and material mechanical properties have also been shown to play a role in determining phenotype [50]. We would argue that the biomaterial cue belongs to the mechano-structural axis because it is intimately bound with the elastic modulus; although if the biomaterial is degradable, it can be dramatically modified by the cells themselves. A number of reviews listing the different biomaterials used in stem cell engineering are available [51-53]. However, very little comparative information can be found regarding the performance and effect of materials on stem cell differentiation, since most approaches use a single material and then test various inducing media to assess the differentiative stimulus provided by the material.

Probably the best studied mechano-structural feature is the elastic modulus or the stiffness of a substrate or a scaffold and its effect on the lineage specification. Engler et al. [50] have pioneered studies on matrix elasticity and its effect on MSCs. Through the use of a well-defined elastically tunable polyacrylamide gel (nominally

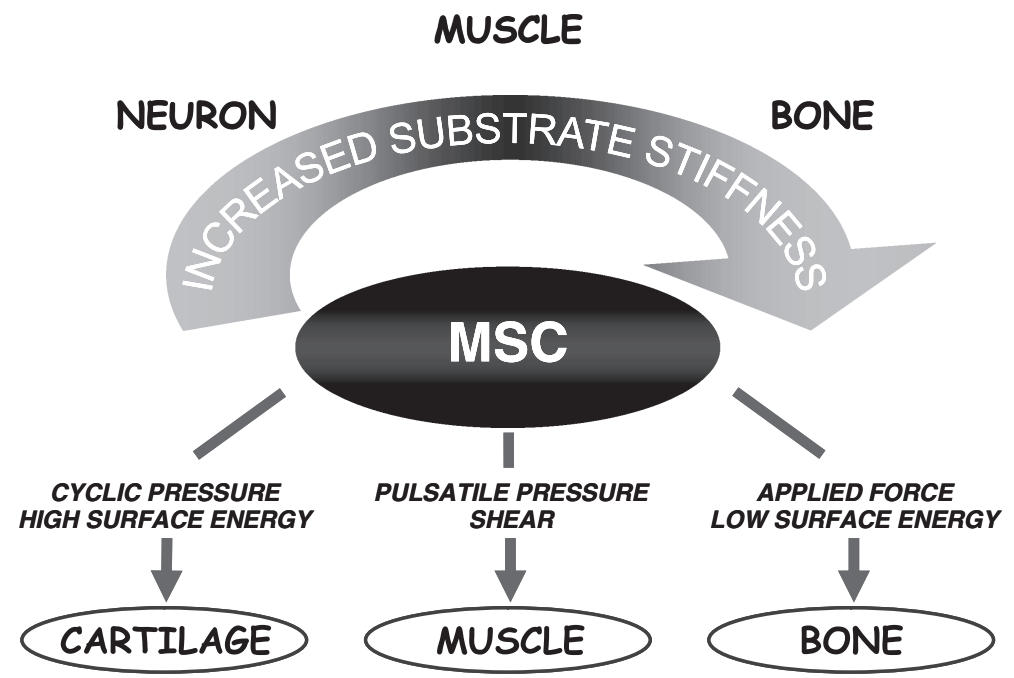

Fig. 7 Some of the factors which influence mesenchymal stem cell differentiation. Very few systematic studies exist, but it is clear that cell substrate interactions, which depend on substrate surface energy and elastic modulus, play a key role in determining stem cell destiny 
2D since cells did not penetrate the cell but adhered on the surface), they observed lineage specification independent of inductive biochemical factors. Softer gels $(0.1-1 \mathrm{kPa})$ were neurogenic, the hardest $(24-40 \mathrm{kPa})$ were osteogenic, while gels with intermediate elastic moduli $(8-17 \mathrm{kPa})$ were myogenic. In all three cases, the elastic modulus matches that of the corresponding native tissue. Figure 7 summarizes the physico-chemical and mechano-structural factors which are known to influence MSC lineage specification and commitment.

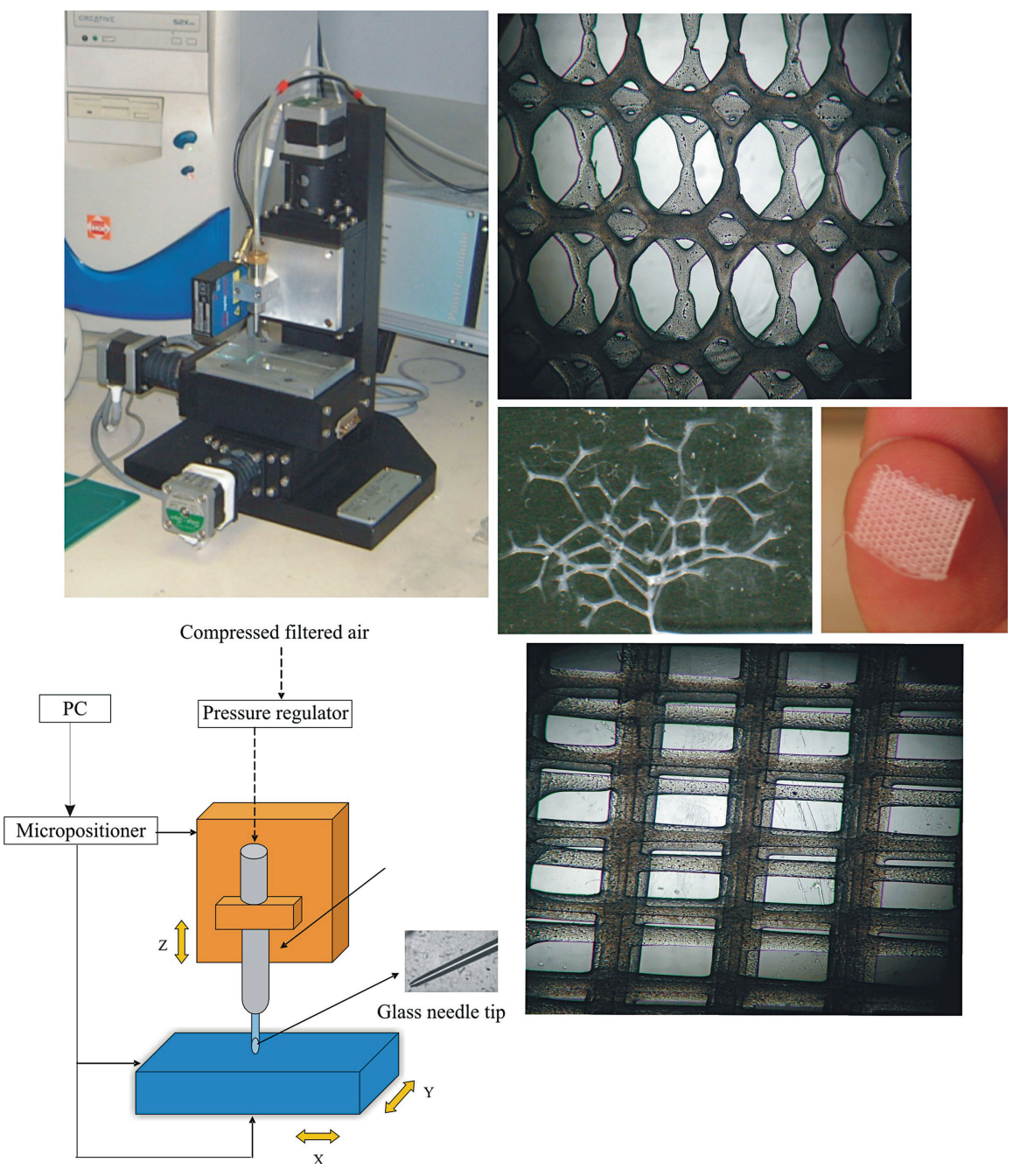

Fig. 8 PAM (pressure-assisted microsyringe) system, its working principle and sample 2D and 3D scaffolds (polylactide and polylactide-co-glycolide). PAM is a CAD/CAM microfabrication method which enables the production of biomaterial scaffolds with tunable geometries and mechanical properties [55] 
The potency of scaffold stiffness and topology in driving cardiac stem cell differentiation in a three-dimensional culture context was confirmed by Forte et al. [54]. Cardiac stem cells adopted the cardiomyocytic phenotype only when cultured in strictly controlled conditions characterized by a critical combination of chemical, biochemical and physical factors, and emulation of the inner myocardial environment. In these studies, the emulation of myocardial environment was achieved by fine-tuning the array of growth factors dissolved in the culture medium and above all the chemistry, topology and stiffness of three-dimensional supports on which stem cells were seeded. The absence of one or more appropriate growth factors or the presence of a polymeric scaffold with stiffness higher than that passively expressed by myocardium did not trigger the differentiating cascade leading to the cardiomyocytic phenotype. Here scaffold stiffness was modulated by changing the topology of the structure using a rapid prototyping technique PAM, illustrated in Fig. 8. The optimal stiffness to induce cardiomyocyte differentiation was in the range $300 \mathrm{kPa}$ on the scaffolds with square pores of about $150 \mu \mathrm{m}$.

Shear stress induced by flow is also an important physical stimulus, particularly for driving cells towards vascular phenotypes. Flow at physiological wall shear stresses typical of blood vessels $(0.1-1.5 \mathrm{~Pa})$ induces differentiation towards endothelial-like characteristics [56-58]. Adamo et al. [59] have recently demonstrated that shear stresses of the order of $0.5 \mathrm{~Pa}$ increase haematopoietic colony-forming potential and expression of haematopoietic markers in mouse embryos. Therefore, fluid shear stresses also influence the differentiation pathways of cells indirectly associated with the vascular system. Like scaffold stiffness, wall shear stress is a parameter which can be easily controlled and modulated using engineering design tools and technology.

\section{Putting It All Together - Making Space for Engineers in Biology}

Many of the factors discussed here have already been studied and identified in adult differentiated cell systems, although several questions are still open to discussion even in this sphere, for example, as concerns cell adhesion in vivo, in vitro and after implantation [60].

Providing these cues in a controlled and coordinated manner in vitro is an enormously challenging task, not currently available in traditional Petri dishes or microwells and cell culture incubators. Indeed, the most commonly cited tools for engineering stem cells are bioreactors [53, 61] and biomaterials [51, 52]. A limiting feature of most of the bioreactor systems described is their narrow range or field of application. Each bioreactor is different and specifically tailored for a single type of tissue and can usually manage only a handful of stimuli.

On the other hand, modelling tools are not considered as belonging to the gantry of methods applied to the study of stem cell fate in vitro. The inherent complexity of the stem cell niche and the differentiative microenvironment and the infinite range of cell responses to different stimuli suggest that stem cell biology and control are 


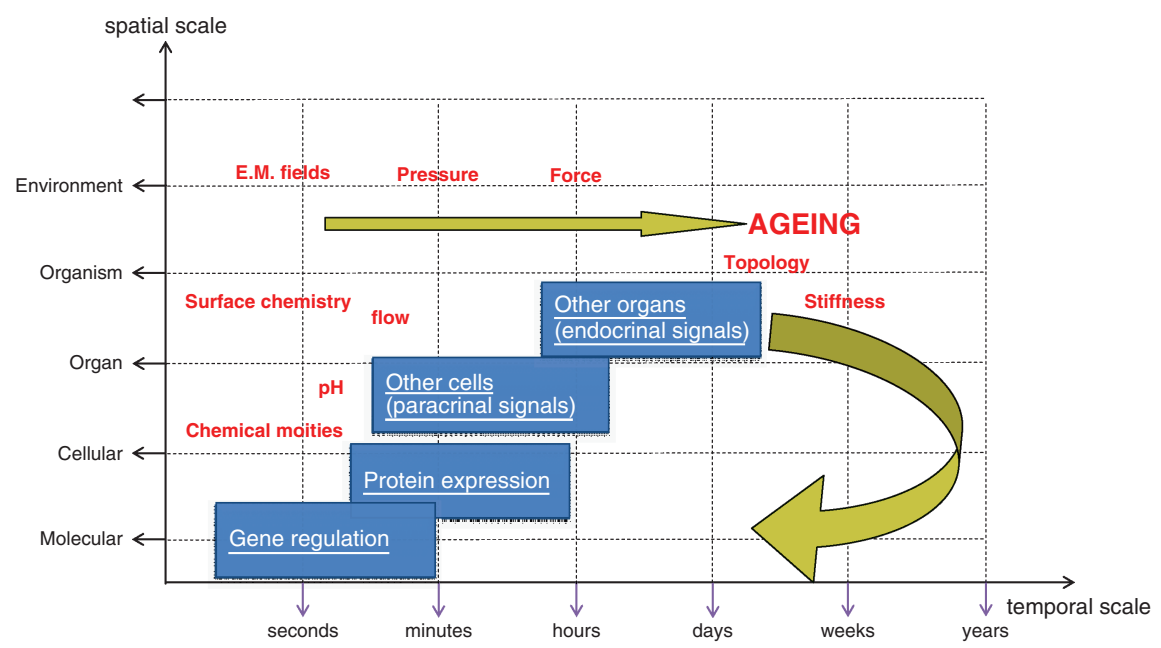

Fig. 9 A multiscale systems biology approach to study the effect of the micro- and macroenvironment of stem cells should take account of the multiple factors which play a role in the scenario and map these using PCA and phase diagrams

ideal candidates for assessment and simulation using a multiscale systems biology approach. The approach consists in coupling network models, be they deterministic or stochastic, across large ranges of length- and timescales to describe complex systems, as described in Fig. 9 [62]. Firstly, we need to single out and identify each parameter and study it individually, in the absence of other factors, or at the very least the other factors should be controlled. Cell response then should be studied at multiple hierarchical levels, genomic, proteomic and metabolomic, followed by a higher system and network level. Large-scale external factors such as flow and pressure can then be linked with molecular factors such as gene expression and biochemical inducers to give a complete picture of the micro- and macroenvironmental framework which orchestrates cell and tissue function.

The cues then need to be combined and appropriate phase diagrams constructed. Alternatively, advanced statistical methods such as three-way PCA (principal component analysis) enable phase maps to be generated from experiments in which multiple cues are present. Since many of the cues or stimuli overlap (such as elastic modulus and strain, surface energy and chemistry) or interact synergically (such as porosity and oxygen supply), this type of analysis could help pinpoint the most crucial factors which drive phenotypic expression in stem cells and their derivatives.

\section{Conclusion}

We are slowly building up a knowledge base on stem cell biology, even though several issues still need to be resolved. Amongst the many obstacles to the clinical application of stem cell technology is the availability of suitable procedures to 
expand the very few multipotent cells present in a tiny tissue sample and to address their fate to create functional portions of tissue.

These goals can be achieved by supplying stem cells with a complex array of biochemical signals and environmental stimuli by means of culture microenvironment with specific and well-controlled physical and chemical factors as well as a suitable three-dimensional environment providing physical support to stem cell adhesion, proliferation and differentiation and geometrical guidance in tissue organization. Together they form what we call here the triaxis of cues: a trio of biochemical, biophysical and biomechanical signalling systems which interact synergically to support both form and function in all living tissues. Providing these cues in a controlled and coordinated manner in vitro is a challenging task, particularly in the case of stem cells, which are known to be inherently unstable within and without their niches. Only a systematic and well-informed approach will bring us closer to understanding the roles of these cues in regulating stem cell fate, providing us with the tools to intervene and control these cells for therapeutic purposes.

\section{References}

1. Ho A. Kinetics and symmetry of divisions of hematopoietic stem cells. Exp Hematol. 2005; 33(1):1-8.

2. Schiffmann Y. Symmetry breaking and convergent extension in early chordate development. Prog Biophys Mol Biol. 2006; 92:209-231.

3. Vandervelde S, van Luyn MJ, Tio RA, Harmsen MC. Signaling factors in stem cell-mediated repair of infarcted myocardium. J Mol Cell Cardiol. 2005; 39:363-376.

4. Rando TA. Stem cells, ageing and the quest for immortality. Nature. 2006; 441:1080.

5. Segers VFM, Lee RT. Stem cell therapy for cardiac disease. Nature. 2008; 451:937.

6. Ho AD, Wagner W, Mahlknecht U. Stem cells and ageing: the potential of stem cells to overcome age-related deteriorations of the body in regenerative medicine. EMBO Rep. 2005; $6: \mathrm{s} 36-\mathrm{s} 38$.

7. Trounson A. The production and directed differentiation of human embryonic stem cells. Endocr Rev. 2006; 27(2):208-219.

8. Kurata H, Guillot PV, Chan J, Nicholas M. Osterix induces osteogenic gene expression but not differentiation in primary human fetal mesenchymal stem cells. Tissue Eng. 2007; 13(7):1512-1523.

9. Bobis S, Jarocha D, Majka M. Mesenchymal stem cells: characteristics and clinical applications. Folia Histochem Cytobiol. 2006; 44(4):215-230.

10. Makino S, Fukuda K, Miyoshi S, Konishi F, Kodama H, Pan J, Sano M, Takahashi T, Hori S, Abe H, Hata J, Umezawa A, Ogawa S. Cardiomyocytes can be generated from marrow stromal cells in vitro. J Clin Invest. 1999 Mar; 103(5):697-705.

11. Jackson KA, Majka SA, Wang H, Pocius J, Craig JH, Majesky MW, Entman ML, Michael LK, Hirschi KK, Goodell MA. Regeneration of ischaemic cardiac muscle and vascular endothelium by adult stem cells. J Clin Invest. 2001; 107:1395-1402.

12. Murry CE, Soonpaa MH, Reinecke H, Nakajima H, Nakajima HO, Rubart M, Pasumarthi KB, Virag JI, Bartelmez SH, Poppa V, Bradford G, Dowell JD, Williams DA, Field LJ. Haematopoietic stem cells do not transdifferentiate into cardiac myocytes in myocardial infarction. Nature. 2004; 428:664-673.

13. Ratajczak MZ, Kucia M, Reca R, Majka M, Janowska-Wieczorek A, Ratajczak Shinji J. Stem cell plasticity revisited: CXCR4-positive cells expressing mRNA for early muscle, liver and neural cells 'hide out' in the bone marrow. Leukemia. 2004; 18:29-40. 
14. Dawn B, Stein AB, Urbanek K, Rota M, Whang B, Rastaldo R, Torella D, Tang XL, Rezazadeh A, Kajstura J, Leri A, Hunt G, Varma J, Prabhu SD, Anversa P, Bolli R. Cardiac stem cells delivered intravascularly traverse the vessel barrier, regenerate infarcted myocardium, and improve cardiac function. Proc Natl Acad Sci USA. 2005; 102:3766-3771.

15. Zampetaki A, Kirton JP, Xu Q. Vascular repair by endothelial progenitor cells. Cardiovasc Res. 2008; 78:413-421.

16. Tian XF, Heng BC, Ge Z, Lu K, Rufaihah AJ, Fan VT, Yeo JF, Cao T. Comparison of osteogenesis of human embryonic stem cells within 2D and 3D culture systems. Scand J Clin Lab Invest. 2008; 68(1):58-67.

17. Park J, Setter V, Wixler V, Schneider H. Umbilical cord blood stem cells: induction of differentiation into mesenchymal lineages by cell-cell contacts with various mesenchymal Cells. Tissue Eng Part A. 2008 15(2):397-406.

18. Ho AD, Wagner W. The beauty of asymmetry: asymmetric divisions and self-renewal in the haematopoietic system. Curr Opin Hematol. 2007; 14(4):330.

19. Mazzei D, Vozzi F, Cisternino A, Vozzi G, Ahluwalia A. A high-throughput bioreactor system for simulating physiological environments. IEEE Trans Ind Electron. 2008; 55:9.

20. McBride SH, Knothe Tate ML. Modulation of stem cell shape and fate A: the role of density and seeding protocol on nucleus shape and gene expression. Tissue Eng Part A. 2008 Sep; 14(9):1561-1572.

21. Bianchi F, Vozzi G, Pescia C, Domenici C, Ahluwalia A. A comparative study of chemical derivatisation methods for spatially differentiated cell adhesion on 2-dimensional microfabricated polymeric matrices. J Biomater Sci Polymer Edn. 2003; 14:1077-1096.

22. Liu X, Lim JY, Donahue HJ, Dhurjati R, Mastro AM, Vogler EM. Influence of substratum surface chemistry/energy and topography on the human fetal osteoblastic cell line hFOB 1.19: phenotypic and genotypic responses observed in vitro. Biomaterials. 2007; 28: 4535-4550.

23. Csete M. Oxygen in the cultivation of stem cells. Ann NY Acad Sci. 2005; 1049:1-8.

24. Studet L, Csete M, Lee SH. Enhanced proliferation, survival, and dopaminergic differentiation of CNS precursors in lowered oxygen. J Neurosci. 2000; 20:7377-7383.

25. Moussavi-Harami F, Duwayri Y, Martin JA, Moussavi-Harami F, Buckwalter JA. Oxygen effects on senescence in chondrocytes and mesenchymal stem cells: consequences for tissue engineering. Iowa Orthop J. 2004; 24:p15-p20.

26. Grayson WL, Zhao F, Izadpanah R, Bunnell B, Ma T. Effects of hypoxia on human mesenchymal stem cell expansion and plasticity in 3D constructs. J Cell Physiol. 2006 May; 207(2):331-339.

27. Fink T, Abildtrup L, Fogd K, Abdallah BM, Kassem M, Ebbesen P, Zachara V. Induction of adipocyte-like phenotype in human mesenchymal stem cells by hypoxia. Stem Cells. 2004; 22:1346-1355.

28. Csete M, Walkikonis J, Slawany N, et al. Oxygen-mediated regulation of skeletal muscle satellite cell proliferation and adipogenesis in culture. J Cell Physiol. 2001; 189:189-196.

29. Lennon DP, Edminson JM, Caplan AI. Cultivation of rat marrow-derived mesenchymal stem cells in reduced oxygen tension: effects on in vitro and in vivo osteochondrogenesis. J Cell Physiol. 2001; 187:345-355.

30. Stolzing A, Sethe S, Scutt A. Stressed stem cells: temperature response in aged mesenchymal stem cells. Stem Cells Dev. 2006; 15:478-487.

31. Stolzing A, Scutt A. Effect of reduced culture temperature on antioxidant defences of mesenchymal stem cells. Free Radic Biol Med. 2006; 41:326-338.

32. Webb K, Haldy V, Tresco PA. Relationships among cell attachment, spreading, cytoskeletal organization, and migration rate for anchorage-dependent cells on model surfaces. J Biomed Mater Res. 2000; 49:362.

33. Curran JM, Chen R, Hunt JA. The guidance of human mesenchymal stem cell differentiation in vitro by controlled modifications to the cell substrate. Biomaterials. 2006 Sep; 27(27):4783-4793. 
34. Radisic M, Park H, Shing H, Consi T, Schoen FJ, Langer R, Freed LE, Vunjak-Novakovic G. Functional assembly of engineered myocardium by electrical stimulation of cardiac myocytes cultured on scaffolds. Proc Natl Acad Sci USA. 2004 Dec 28; 101(52):18129-18134.

35. Sauer H, Rahimi G, Hescheler J, Wartenberg M. Effects of electrical fields on cardiomyocyte differentiation of embryonic stem cells. J Cell Biochem. 1999 Dec 15; 75(4):710-723.

36. Sauer H, Bekhite MM, Hescheler J, Wartenberg M. Redox control of angiogenic factors and CD31-positive vessel-like structures in mouse embryonic stem cells after direct current electrical field stimulation. Exp Cell Res. 2005 Apr 1; 304(2):380-390.

37. Abilez O, Benharash P, Miyamoto E, Gale A, Xu C, Zarins CK. P19 progenitor cells progress to organized contracting myocytes after chemical and electrical stimulation: implications for vascular tissue engineering. Endovasc Ther. 2006 June; 13(3):377-388.

38. Serena E, Flaibani M, Carnio S, Boldrin L, Vitiello L, De Coppi P, Elvassore N. Electrophysiologic stimulation improves myogenic potential of muscle precursor cells grown in a 3D collagen scaffold. Neurol Res. 2008 Mar; 30(2):207-214.

39. Genovese JA, Spadaccio C, Langer J, Habe J, Jackson J, Patel AN. Electrostimulation induces cardiomyocyte predifferentiation of fibroblasts. Biochem Biophys Res Commun. 2008 Jun 6; 370(3):450-455.

40. Yamaguchi DT, Huang J, Ma D, Wang PK. Inhibition of gap junction intercellular communication by extremely low-frequency electromagnetic fields in osteoblast-like models is dependent on cell differentiation. J Cell Physiol. 2002 Feb; 190(2):180-188.

41. Van Den Heuvel R, Leppens H, Nêmethova G, Verschaeve L. Haematopoietic cell proliferation in murine bone marrow cells exposed to extreme low frequency (ELF) electromagnetic fields. Toxicol In Vitro. 2001 Aug-Oct; 15(4-5):351-355.

42. Czyz J, Nikolova T, Schuderer J, Kuster N, Wobus AM. Non-thermal effects of power-line magnetic fields $(50 \mathrm{~Hz})$ on gene expression levels of pluripotent embryonic stem cells-the role of tumour suppressor p53. Mutat Res. 2004 Jan 10; 557(1):63-74.

43. Ebisawa K, Hata K, Okada K, Kimata K, Ueda M, Torii S, Watanabe H. Ultrasound enhances transforming growth factor beta-mediated chondrocyte differentiation of human mesenchymal stem cells. Tissue Eng. 2004 May-Jun; 10(5-6):921-929.

44. Cui JH, Park SR, Park K, Choi BH, Min BH. Preconditioning of mesenchymal stem cells with low-intensity ultrasound for cartilage formation in vivo. Tissue Eng. 2007 Feb; 13(2): 351-360.

45. Baharvand H, Hashemi SM, Ashtiani SM, Farrokhi A. Differentiation of human embryonic stem cells into hepatocytes in 2D and 3D culture systems in vitro. Int J Dev Biol. 2006; 50:645-652.

46. Brännvall K, Bergman K, Wallenquist U, Svahn S, Bowden T, Hilborn J, ForsbergNilsson K. Enhanced neuronal differentiation in a three-dimensional collagen-hyaluronan matrix. J Neurosci Res. 2007 Aug 1; 85(10):2138-2146.

47. Levenberg S, Huang NF, Lavik E, Rogers AB, Itskovitz-Eldor J, Langer R. Differentiation of human embryonic stem cells on three-dimensional polymer scaffolds. PNAS. 2003; 100:12741-12746.

48. Liu H, Roy K. Biomimetic three-dimensional cultures significantly increase hematopoietic differentiation efficacy of embryonic stem cells. Tissue Eng. 2005 Jan; 11(1-2):319-330.

49. Liu H, Lin J, Roy K. Effect of 3D scaffold and dynamic culture condition on the global gene expression profile of mouse embryonic stem cells. Biomaterials. 2006; 27:5978-5989.

50. Engler AJ, Sen S, Sweeney HL, Disher DE. Matrix elasticity directs stem cell lineage specification. Cell. 2006; 126:677-689.

51. Saha K, Pollock JF, Schaffer DV, Healy KE. Designing synthetic materials to control stem cell phenotype. Curr Opin Chem Biol. 2007; 11:381-387.

52. Hwang NS, Varghese S, Elisseeff J. Controlled differentiation of stem cells. Adv Drug Deliv Rev. 2008; 60:199-214.

53. Burdick JA, Vunjak-Novakovic G. Review: engineered microenvironments for controlled stem cell differentiation. Tissue Eng A. 2008; 14:1809-1820. 
54. Forte G, Carotenuto F, Pagliari F, Pagliari S, Cossa P, Fiaccavento R, Ahluwalia A, Vozzi G, Vinci B, Serafino A, Rinaldi A, Traversa E, Carosella L, Minieri M, Di Nardo P. Criticality of the biological and physical stimuli array inducing resident cardiac stem cell determination. Stem Cells. 2008 May; 26(8):2093-2103.

55. Mariani M, Rosatini F, Vozzi G, Previti A, Ahluwalia A. Characterisation of tissue engineering scaffolds microfabricated with PAM. Tissue Eng. 2006; 12(3):547-558.

56. Wang H, Riha GM, Yan S, Li M, Chai H, Yang H, Yao Q, Chen C. Shear stress induces endothelial differentiation from a murine embryonic mesenchymal progenitor cell line. Arterioscler Thromb Vasc Biol. 2005; 25:1817-1823.

57. Metallo CM, Vodyanik MA, de Pablo JJ, Slukvin II, Palecek SP. The response of human embryonic stem cell-derived endothelial cells to shear stress. Biotechnol Bioeng. 2008 July 1; 100(4):830-837.

58. Wu CC, Chao YC, Chen CN, Chien S, Chen YC, Chien CC, Chiu JJ, Linju Yen B. Synergism of biochemical and mechanical stimuli in the differentiation of human placenta-derived multipotent cells into endothelial cells. J Biomech. 2008; 41(4):813-821.

59. Adamo L, Naveiras O, Wenzel PL, McKinney-Freeman S, Mack PJ, Gracia-Sancho J, Suchy-Dicey A, Yoshimoto M, Lensch MW, Yoder MC, García-Cardeñ G, Daley GQ. Biomechanical forces promote embryonic haematopoiesis. Nature. 2009; doi:10.1038/ nature08073.

60. Wilson CJ, Clegg RE, Leavesley DI, Pearcy MJ. Mediation of biomaterial-cell interactions by adsorbed proteins: a review. Tissue Eng. 2005 Jan-Feb; 11(1-2):1-18.

61. Godara P, McFarland CD, Nordon RE. Design of bioreactors for mesenchymal stem cell tissue engineering. J Chem Technol Biotechnol. 2008; 83:408-420.

62. Evans DJW, Lawford PV, Gunn J, et al. The application of multiscale modelling to the process of development and prevention of stenosis in a stented coronary artery. Philos Trans R Soc A. 2008; 366:3343-3360. 
黛 Springer

http://www.springer.com/978-3-642-11864-7

Stem Cell Engineering

Principles and Applications

(Eds.) G.M. Artmann; S. Minger; J. Hescheler

2011, XLI, 547 p. 153 illus., Hardcover

ISBN: $978-3-642-11864-7$ 